\title{
Short Communication: Augmentation of cardioprotective effect of captopril by Costus speciosus against isoproterenol induced myocardial toxicity in rats
}

\author{
ABDULRAHMAN A.I. AL-YAHYA ${ }^{1, \bullet}$, MOHAMMED ASAD ${ }^{1}$, ABDULMONIEM SADABY ${ }^{1}$, \\ KHALID ELFAKKI IBRAHIM ${ }^{2}$ \\ ${ }^{1}$ College of Applied Medical Sciences, Shaqra University. P O Box 1383, Shaqra 11961, Saudi Arabia, Tel.: +966-531339880, \\ `email: alya7ya@su.edu.sa, alyahyashaqra@gmail.com \\ ${ }^{2}$ Department of Zoology, College of Science, King Saud University. Riyadh 12371, Saudi Arabia
}

Manuscript received: 12 July 2017. Revision accepted: 21 August 2017.

\begin{abstract}
Al-Yahya AAI, Asad M, Sadaby A, Ibrahim KE. 2017. Short Communication: Augmentation of cardioprotective effect of captopril by Costus speciosus against isoproterenol induced myocardial toxicity in rats. Nusantara Bioscience 9: 295-299. This study determined the pharmacodynamic interaction of captopril, a known cardioprotective effect with methanolic extract of Costus speciosus rhizomes (Costaceae) that is known for its powerful antioxidant action during isoproterenol induced cardiac toxicity in rats. A methanolic extract of the rhizomes was prepared by maceration. Rats were administered the methanolic extract at two different doses of $200 \mathrm{mg} / \mathrm{k}$ or $400 \mathrm{mg} / \mathrm{kg}$ orally and captopril was administered orally at a dose of $30 \mathrm{mg} / \mathrm{kg}$. All the drugs alone or in combination were once daily for two weeks. At the end of treatment period, two doses of isoproterenol $(150 \mathrm{mg} / \mathrm{kg}$, s.c) were administered to rats at $24 \mathrm{hr}$ interval. Blood was withdrawn to estimate creatinine kinase-MB (CK-MB) activities. The heart tissue was subjected to histological examinations to determine the extent of damage. Isoproterenol induced severe damage to the myocardium that was indicated through an elevation in serum CK-MB activity and the same was confirmed by histological examinations. Costus speciosus at both the tested doses attenuated the damage produced by isoproterenol. Both the doses caused a decrease in the biomarker activity as well as reduced the myocardial damage as observed in histological examination. A similar effect was observed with captopril. The co-administration of captopril with either dose of Costus speciosus demonstrated excellent cardioprotection suggesting that combination of this herb with captopril augments its cardioprotective action. It was concluded that Costus speciosus shows dose-dependent cardioprotection and augments the cardioprotective effect of captopril during isoproterenol induced cardiotoxicity in rats.
\end{abstract}

Keywords: Acute myocardial injury, Costus speciosus extract, captopril, isoproterenol, interaction, methanolic extract

\section{INTRODUCTION}

Costus speciosus (Koen ex. Retz.) belongs to the family Costaceae (Zingiberaceae). The plant possesses many pharmacological activities and is used traditionally in the treatment of several diseases. The plant is so widely used that is considered as endangered if its use continues to exceed its cultivation. Some of the pharmacological activities reported for this plant includes anti-inflammatory, antiangiogenic, antioxidant, antibacterial and antifungal effects due to presence of susquiterpines (Duraipandiyan et al. 2012; Al-Attas et al. 2015; Selim and Al Jaouni 2016), antihyperglycemic activity that is mediated through inhibitory effect on $\alpha$-glucosidase and glycation (Perera et al. 2016), anticholinesterase (Bhattacharya et al. 1972), antipyretic, anti-inflammatory and analgesic activities (Srivastava et al. 2013) among other activities that includes antidiuretic, larvicidal, antistress, estrogenic, astringent, aphrodisiac, purgative, anthelminthic, expectorant activities. It also has anti-fertility, anabolic properties (Pawar and Pawar 2012).

Of all these activities of Costus speciosus, the most interesting and widely studied effect is its antioxidant effect. Though many plants are known to have antioxidant action, the rhizomes is believed to have very strong antioxidant effect that can attenuate the Fenton reactionmediated oxidation of biological lipid substrates (Pai Kotebagilu et al. 2015). It was rated as third plant with potent antioxidant effect among eighteen commonly used antioxidant plants (Lee et al. 2015). It is also reported to reduce hydrogen peroxide induced oxidative damage on biological substrates (Pai Kotebagilu et al. 2014) and also prevent development of cancer due to its antioxidant action (Baskar et al. 2012). The antioxidant effect of this plant is directly related to its phenolic contents (Vijayalakshmi and Sarada 2008; Nehete et al. 2010).

It is well known from several studies that plants having phenolic compounds and exhibiting antioxidant effects protects heart from different types of damage such as ischemia reperfusion and isoproterenol induced cardiac injury (Panda et al. 2016; Wang et al. 2017; Cao et al. 2017; Garjani et al. 2017; Rasines-Perea et al. 2017). Furthermore, the rhizomes of Costus speciosus known as $R u-r t a$ are believed to regulate blood pressure and correct heart problems (Clifford 2001). Hence, we studied earlier its cardioprotective effect and demonstrated that it has good cardioprotective action (unpublished data). However, the trend of using herbal plants alone is declining and use of 
herbal plants as supplements along with modern medicine is on the rise. Hence, the present study was carried out to determine if the coadministration of methanolic extract of rhizomes of Costus speciosus can augment the cardioprotective effect of captopril on myocardial damage induced by isoproterenol in rats.

\section{MATERIALS AND METHODS}

\section{Preparation of the extract}

The rhizomes of Costus speciosus were purchased from a local market in Riyadh, Saudi Arabia. It was authenticated by Prof A.M. Sadaby (College of Applied Medical Sciences, Shaqra University, Saudi Arabia) and a voucher specimen (CAMS/rh03/09-2016) has been preserved in the college for future reference. The rhizomes were finely powdered and extracted using methanol in a closed glass jar for $72 \mathrm{hr}$ initially. The extraction of the solid residue (marc) was further done twice for $48 \mathrm{hr}$ each. The extract obtained was evaporated under reduced pressure. The final extract obtained after evaporation was stored in a refrigerator till use.

\section{Preliminary chemical analysis}

The preliminary chemical analysis revealed the presence of carbohydrates, saponins, flavovoids and amino acids.

\section{Experimental animals}

Laboratory bred male Wistar rats (180-220 g) were housed in a controlled environment at a temperature of 25 $\pm 2{ }^{\circ} \mathrm{C}$ under 12: $12 \mathrm{hr}$ light dark cycle. The animals were maintained under standard conditions in an animal house and all the experimental procedures were approved by the university scientific committee.

\section{Dose selection}

The methanolic extract of Costus speciosus was administered at two doses of $200 \mathrm{mg} / \mathrm{kg}$ and $400 \mathrm{mg} / \mathrm{kg}$ orally. The doses were selected after doing preliminary experiments in our laboratory and from previous reports (Ali et al. 2014). Captopril was administered at a dose of $30 \mathrm{mg} / \mathrm{kg}$ orally (Milanez et al. 1997). The extract and captopril were suspended in distilled water using $2 \%$ acacia. The control animals received only vehicle, which as $2 \%$ acacia in water.

\section{Isoproterenol induced myocardial damage in rats}

Animals were divided into seven groups of six animals each, i.e. (i) Group I had normal animals and they received only vehicle $(2 \mathrm{ml} / \mathrm{kg}, p . o)$. (ii) Group II animals were treated with vehicle $(2 \mathrm{ml} / \mathrm{kg}$, p.o $)$ for two weeks and they received isoproterenol (ISO). (iii) Groups III and IV received orally Costus speciosus extract- $200 \mathrm{mg} / \mathrm{kg}$ and $400 \mathrm{mg} / \mathrm{kg}$, respectively for two weeks and followed by administration of ISO. (iv) Group $\mathrm{V}$ was treated with captopril at a dose of $30 \mathrm{mg} / \mathrm{kg}$ orally for two weeks followed by ISO. (v) Group VI and VII received Costus speciosus extract- $200 \mathrm{mg} / \mathrm{kg}$ and $400 \mathrm{mg} / \mathrm{kg}$, along with captopril $(30 \mathrm{mg} / \mathrm{kg})$ respectively for two weeks.

All treatment was given daily for 2 weeks. At the end of the treatment period, ISO $(150 \mathrm{mg} / \mathrm{kg}$, s.c $)$ was administered to all the animals (except group I) for two consecutive days (Asdaq et al. 2008). Forty eight hours after the first dose of ISO, animals were anesthetized using ether and blood was withdrawn and the serum was used for the estimation of creatinine kinase-MB (CK-MB) using commercially available kits. Thereafter, all the animals were sacrificed, and the hearts were used for histological examinations using $H \& E$ stain. The myocardial damage was determined by giving scores depending on the intensity as follows (Karthikeyan et al. 2007); no changes - score 00; mild-score 01 (focal myocytes damage or small multifocal degeneration with slight degree of inflammatory process); moderate-score 02 (extensive myofibrillar degeneration and/or diffuse inflammatory process); marked-score 03 (necrosis with diffuse inflammatory process).

\section{Statistical analysis}

Results are given as mean \pm SEM. Statistical significance was determined through one-way analysis of variance (ANOVA) followed by Tukey-Kramer multiple comparison tests. For comparison of histological scores, Kruskal-Wallis test with Dunn post test was used. $P<0.05$ indicated statistically significant difference.

\section{RESULTS AND DISCUSSION}

\section{Effect on serum CK-MB levels}

Administration of ISO to rats induced myocardial damage that was confirmed by an increase in serum CKMB levels compared to normal animals. The methanolic extract of Costus speciosus significantly reduced the serum CK-MB levels in a dose dependent manner when compared to ISO administered control group. $(p<0.001)$. As expected, captopril $(30 \mathrm{mg} / \mathrm{kg})$ also reduced the serum CKMB levels when compared to ISO control group $(p<0.001)$ indicating cardioprotective effect. The coadministration of either dose of methanolic extract of Costus speciosus with captopril (30 mg/kg) showed the maximum effect (Figure 1).

\section{Effect on histological scores}

Heart damage was determined by loss of cellular architecture, nuclear duplication, increased infiltration of leucocytes and prominent hyperchromasia. These changes were prominent in control animals treated with ISO (Figure 3 ). The histological scores that were given based on the severity of damage was significantly less in Costus speciosus (400 mg/kg, p.o) and captopril (30 mg/kg, p.o) treated groups compared to ISO treated control while the lower dose of Costus speciosus (200 mg/kg, p.o) did not show any significant reduction in severity scores (Figure 2). Sections of heart tissue from animals treated with lower dose of Costus speciosus (200 mg/kg, p.o) showed mild to moderate damage ranging from loss of cellular architecture 
to nuclear duplication and increased infiltration of leucocytes (Figure 4) while those treated with higher dose of Costus speciosus $(400 \mathrm{mg} / \mathrm{kg}$, p.o) showed no or very mild damage such as some loss of cellular architecture (Figure 5). Captopril (30 mg/kg, p.o) almost prevented the ISO induced myocardial damage with sections from animals showing almost no damage (Figure 6) while the maximum effect was observed in animals treated with either dose of Costus speciosus plus captopril (Figure 7).

\section{Discussion}

The present study was undertaken to determine the interaction of methanolic extract of Costus speciosus rhizomes with captopril on the ISO induced myocardial infarction in rats. The result of the study suggests that Costus speciosus rhizomes augment the cardioprotective activity of captopril during myocardial damage induced by ISO in rats.

As mentioned earlier, Costus speciosus is so widely used traditionally that it is on the verge of extinction if its cultivation is not increased indicating its therapeutic potential. As mentioned earlier, the rhizomes of Costus speciosus are the most widely part of the plant; hence, this was used in the present study. The doses of the extract for administration to rats were selected in earlier literature (Ali et al. 2014) and also by preliminary studies carried out in our laboratory. In our study, we observed that administration of higher doses of extract such as $500 \mathrm{mg} / \mathrm{kg}$ or $1000 \mathrm{mg} / \mathrm{kg}$ orally produced toxic effects in the animals. The animals started to show weakness after 4-5 days of drug administration and continuing the administration for 3-4 days more resulted in deaths of some animals. Hence, doses more than $400 \mathrm{mg} / \mathrm{kg}$ orally were not used. On the other hand, administration of doses less than $200 \mathrm{mg} / \mathrm{kg}$ such as $100 \mathrm{mg} / \mathrm{kg}$ or $50 \mathrm{mg} / \mathrm{kg}$ did not produce any significant effect.

The rhizomes of Costus speciosus are reported to contain a number of chemical constituents that includes saponin such as tigogenin, diosgenin, sapogenin along with steroids and alkaloids (Muniyandi et al. 2013). It is also known to contain aliphatic hydroxyl ketones, triterpenes, starch mucilage, oxa-acids, fatty acids, abscisic acid and corticosteroids (Rajesh et al. 2009). An important constituent with respect to the antioxidant effect of Costus speciosus rhizomes are flavonoids, that are a subclass of polyphenols, which are further divided into subclasses such as flavones, flavonols, isoflavones, anthocyanins, flavanols, proanthocyanidins and other plant phenolics (Nehete et al. 2010; Chang et al. 2012). As mentioned earlier (Vijayalakshmi and Sarada, 2008; Nehete et al. 2010), these polyphenols are responsible for the antioxidant effect of Costus speciosus.

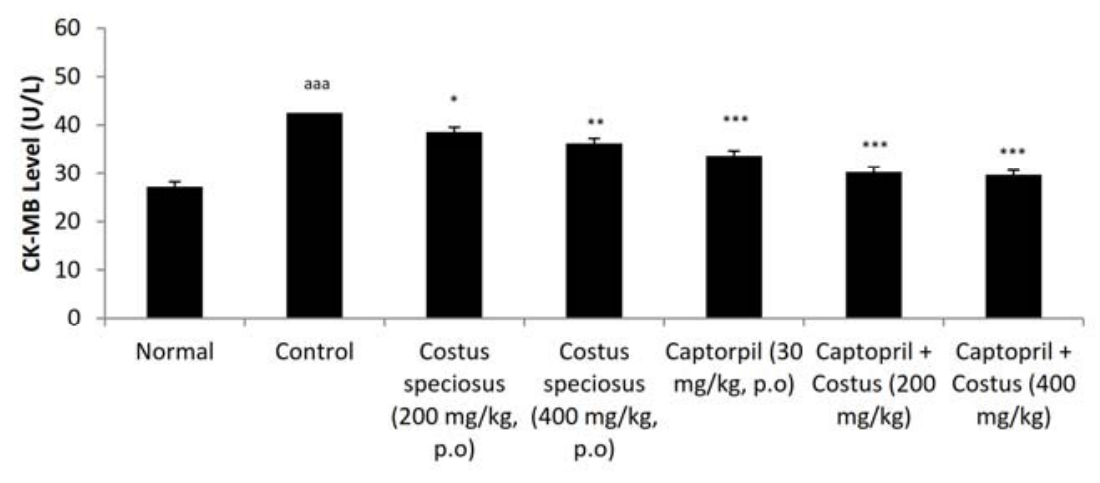

Figure 1: Effect of Costus speciosus extract and captopril on serum CK-MB levels. Note: All values are mean $\pm \mathrm{SEM}, \mathrm{n}=6$, ${ }^{\text {aaa }} p<0.001$ compared to normal animals, ${ }^{*} p<0.05,{ }^{* *} p<0.01,{ }^{* * *} p<0.001$ compared to ISO treated control

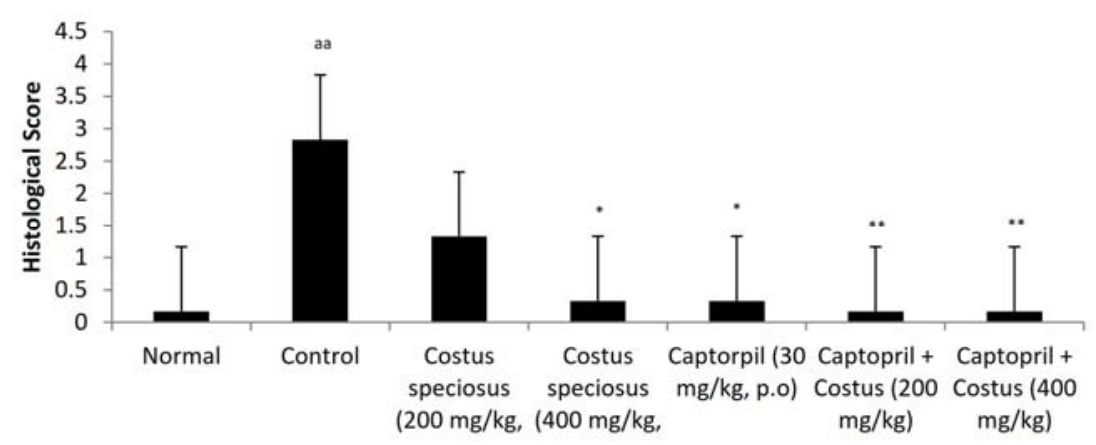

Figure 2: Effect of Costus speciosus extract and captopril on histological scores. Note: All values are mean $\pm \mathrm{SEM}, \mathrm{n}=6,{ }^{\text {aa }} p<0.01$ compared to normal animals, ${ }^{*} p<0.05,{ }^{* *} p<0.01,{ }^{* * *} p<0.001$ compared to ISO treated control 


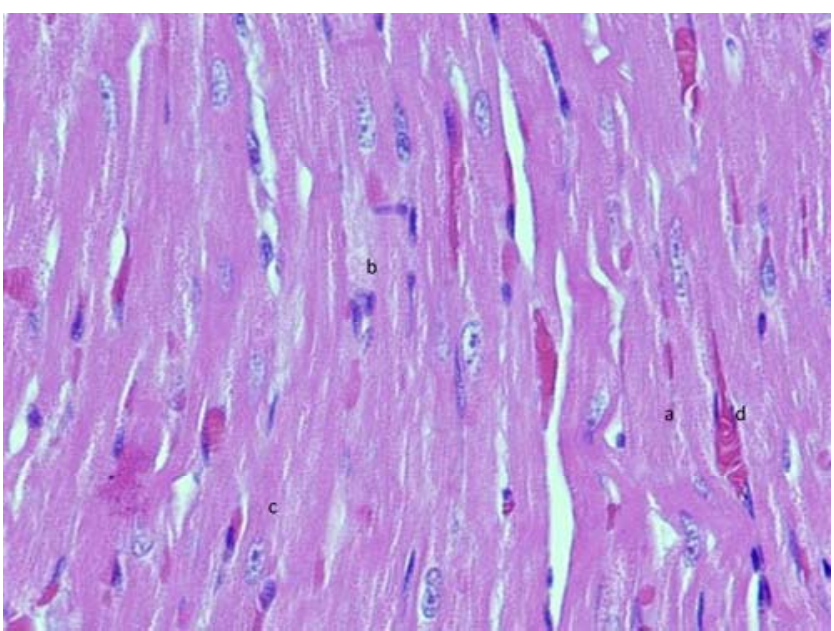

Figure 3: Microscopic section of heart from isoproterenol (ISO) control animals (H\&E stained, $\times 400)$ showing severe damage to heart tissue. There is loss of cellular architecture (a), nuclear duplication (b) , increased infiltration of leucocytes (c) and prominent hyperchromasia (d).

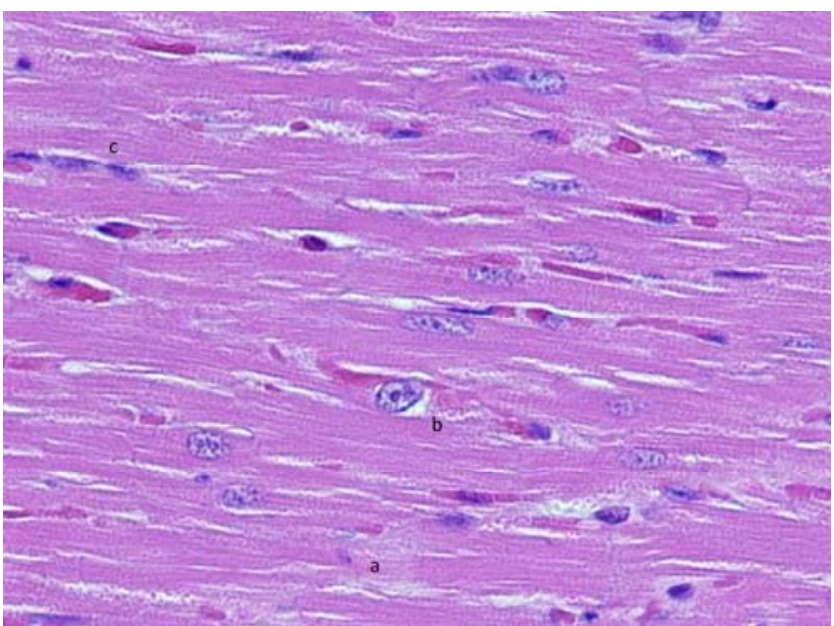

Figure 4: Microscopic section of heart from Costus speciosus $(200 \mathrm{mg} / \mathrm{kg}$, p.o $)+$ isoproterenol (ISO) treated animals (H\&E stained, $\times 400)$. . There is loss of cellular architecture (a), nuclear duplication (b) and increased infiltration of leucocytes (c)

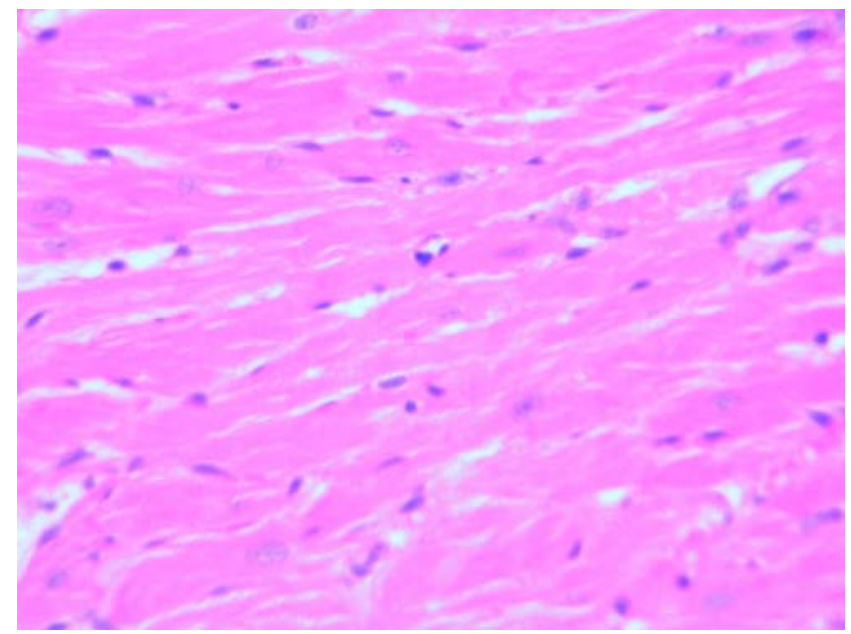

Figure 5: Microscopic section of heart from Costus speciosus $(400 \mathrm{mg} / \mathrm{kg}$, p.o $)+$ isoproterenol (ISO) treated animals (H\&E stained, $\times 400$ ) showing almost normal cytoarchitechture.

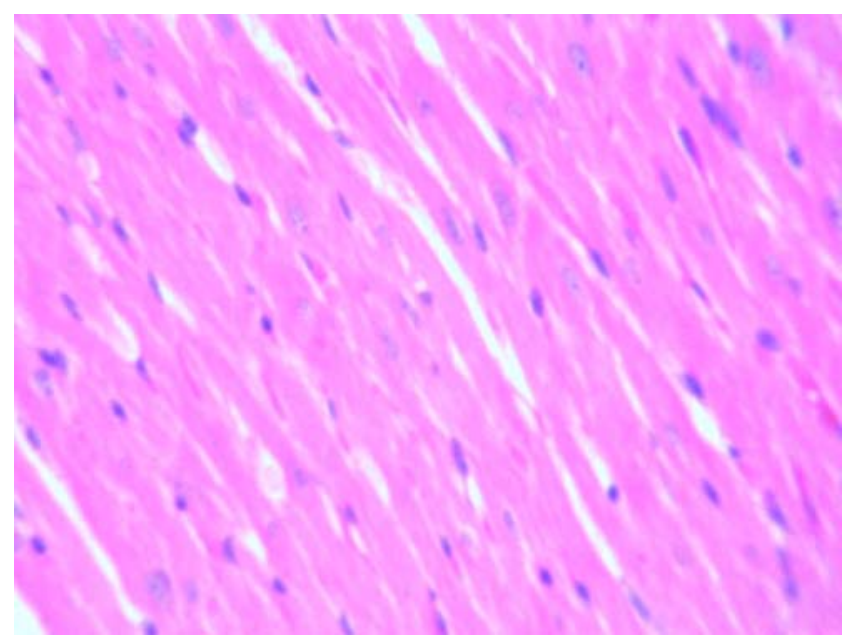

Figure 6: Microscopic section of heart from captopril $(30 \mathrm{mg} / \mathrm{kg}$, p.o) + isoproterenol (ISO) treated animals (H\&E stained, $\times 400)$ showing almost normal cytoarchitechture

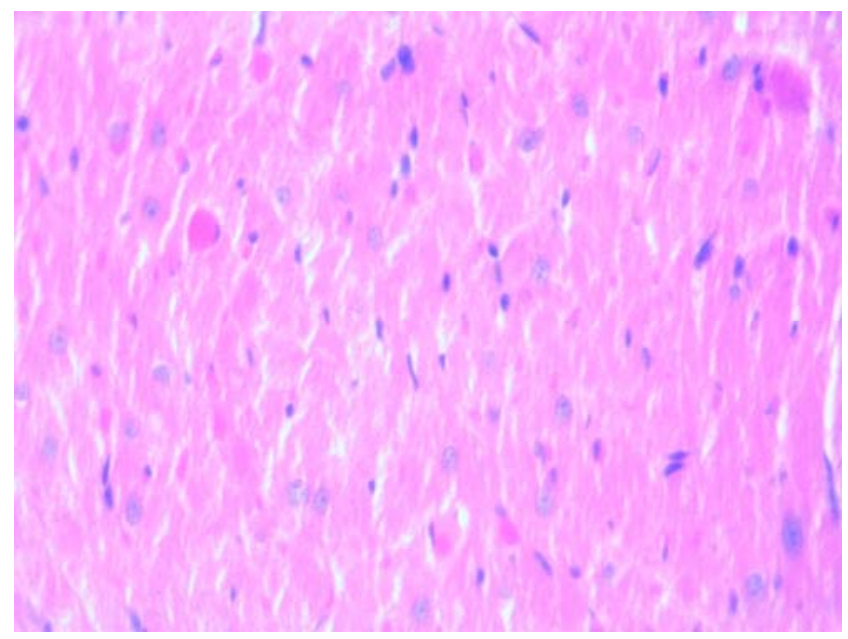

Figure 7: Microscopic section of heart from Costus speciosus $(400 \mathrm{mg} / \mathrm{kg}$, p.o $)+$ captopril $(30 \mathrm{mg} / \mathrm{kg}$, p.o $)+$ isoproterenol (ISO) treated animals (H\&E stained, $\times 400)$ showing almost normal cytoarchitechture.

ISO induces myocardial through reduction of endogenous antioxidants such as superoxide dismutase (SOD) and catalase which leads to myocardial damage due to oxidative stress (Yang et al. 2011). Once the myocardium is damages due to oxidative stress, it causes release of CK-MB present in the myocytes to the plasma leading to an increase in serum CK-MB levels. An increase in levels of CK-MB is an indication of myocardial damage. In the present study, ISO administration led to an increase in serum CK-MB levels indicating damage to myocardium. Administration of methanolic extract of Costus speciosus rhizomes and captopril attenuated the increase in serum CK-MB levels suggesting cardioprotective effect. The cardioprotective effect was further confirmed by histological studies, wherein changes in cellular architecture inflammation and necrosis were taken as 
parameters for determination of myocardial damage. The results of the histological studies supported the biochemical findings.

The exact mechanism for augmentation of cardioprotective effect of captopril by Costus speciosus rhizomes cannot be explained with the present data. However, we speculate that the powerful antioxidant effect of the plant may contribute at least in part to this augmentative cardioprotective action. However, a detailed pharmacokinetic study is also required to determine the effect of this extract on the pharmacokinetics of captopril.

To conclude, the methanolic extract of Costus speciosus rhizomes possess good cardioprotective effect and augments the cardioprotective effect of captopril against isoproterenol induced myocardial damage in rats.

\section{REFERENCES}

Al-Attas AA, El-Shaer NS, Mohamed GA, Ibrahim SR, Esmat A. 2015 Anti-inflammatory sesquiterpenes from Costus speciosus rhizomes. J Ethnopharmacol 176: 365-74.

Ali HA, Almaghrabi OA, Afifi ME. 2014. Molecular mechanisms of antihyperglycemic effects of Costus speciosus extract in streptozotocininduced diabetic rats. Saudi Med J 35 (12): 1501-1506.

Asdaq SM, Inamdar MN, Asad M, Nanjundan PK. 2008. Interaction of propranolol with garlic in isoproterenol induced myocardial infarction in rat. J Pharmacol Toxicol 3: 414-424.

Baskar AA, Al Numair KS, Alsaif MA, Ignacimuthu S. 2012. In vitro antioxidant and antiproliferative potential of medicinal plants used in traditional Indian medicine to treat cancer. Redox Rep 17 (4): 145 156.

Bhattacharya S.K, Parik A.K, Debnath P.K, Pandey V.B, Neogy N.C. 1972. Anticholinesterase activity of Costus speciosus alkaloids. Indian J. Pharmacol 4: 178-187.

Cao YG, Zheng XK, Yang FF, Li F, Qi M, Zhang YL, Zhao X, Kuang HX, Feng WS. 2017. Two new phenolic constituents from the root bark of Morus alba L. and their cardioprotective activity. Nat Prod Res 28: 1-8.

Chang YQ, Tan SN, Yong JWH Ge L. 2012. Determination of flavonoids in Costus speciosus and Etlingera elatior by liquid chromatography mass spectrometry. Anal Lett 45: 345-355.

Clifford T. 2001. Tibetan Buddhist medicine and psychiatry. Motilal Banarsidas Publishers Private Limited New Delhi, India.

Duraipandiyan V, Al-Harbi NA, Ignacimuthu S, Muthukumar C. 2012. Antimicrobial activity of sesquiterpene lactones isolated from traditional medicinal plant, Costus speciosus (Koen ex.Retz.) Sm. BMC Compl Altern Med 12: 13. DOI: 10.1186/1472-6882-12-13.

Garjani A, Tila D, Hamedeyazdan S, Vaez H, Rameshrad M, Pashaii M, Fathiazad F. 2017. An investigation on cardioprotective potential of Marrubium vulgare aqueous fraction against ischemia-reperfusion injury in isolated rat heart. Folia Morphol (Warsz). DOI: 10.5603/FM.a2017.0011.

Karthikeyan K, SaralaBai BR, Devaraj N. 2007. Cardioprotective effect of grape seed proanthocyanidins on isoproterenol-induced myocardial injury in rats. Intl J Cardiol 115: 326-333.

Lee YH, Choo C, Watawana MI, Jayawardena N, Waisundara VY. 2015. An appraisal of eighteen commonly consumed edible plants as functional food based on their antioxidant and starch hydrolase inhibitory activities. J Sci Food Agric 95 (14): 2956-964.

Milanez MC, Gomes MG, Vassallo DV, Mill JG. 1997. Effects of captopril on interstitial collagen in the myocardium after infarction in rats. J Card Fail 3: 189-197.

Muniyandi SK, Nandanan AT, Veeti SC, Narayanan A, Ganesan B. 2013. Studies on Costus speciosus koen alcoholic extract for larvicidal activity. Inter J Pharmacog Phytochem Res 5 (4): 328-329.

Nehete J, Bhatia M, Narkhede M. 2010. In-vitro evaluation of antioxidant activity and phenolic content of Costus speciosus (koen) J.E. Sm. Iran J Pharm Res 9 (3): 271-277.

Pai Kotebagilu N, Reddy Palvai V, Urooj A. 2014. Protective Effect of Selected Medicinal Plants against Hydrogen Peroxide Induced Oxidative Damage on Biological Substrates. Int J Med Chem 2014: ID861084. DOI: 10.1155/2014/861084.

Pai Kotebagilu N, Reddy Palvai V, Urooj A. 2015. Ex-vivo antioxidant activity of selected medicinal plants against fenton reaction-mediated oxidation of biological lipid substrates. Biochem Res Intl 2015: ID728621. DOI: 10.1155/2015/728621.

Panda V, Laddha A, Nandave M, Srinath S. 2016. Dietary phenolic acids of macrotyloma uniflorum (horse gram) protect the rat heart against isoproterenol-induced myocardial infarction. Phytother Res 30 (7): 1146-155.

Pawar VA, Pawar PR. 2012. Costus speciosus: an important medicinal plant. Inter J Sci Res 3 (7): 28-33.

Perera HK, Premadasa WK, Poongunran J. 2016. $\alpha$-glucosidase and glycation inhibitory effects of Costus speciosus leaves. BMC Compl Altern Med 16: 2. DOI: 10.1186/s12906-015-0982-z.

Rajesh MS, Harish MS, Sathyaprakash RJ, Shetty AR, Shivananda TN. 2009. Antihyperglycemic activity of the various extracts of Costus speciosus rhizomes. J Nat Rem 9 (2): 235-241.

Rasines-Perea Z, Teissedre PL. 2017. Grape Polyphenols' Effects in human cardiovascular diseases and diabetes. Molecules 22 (1): 26-36.

Selim S, Al Jaouni S. 2016. Anti-inflammatory, antioxidant and antiangiogenic activities of diosgenin isolated from traditional medicinal plant, Costus speciosus (Koen ex.Retz.) Sm. Nat Prod Res 30 (16): 1830-1833.

Srivastava S, Singh P, Jha KK, Mishra G, Srivastava S, Khosa RL. 2013. Antiinflammatory, analgesic and antipyretic activities of aerial parts of Costus speciosus Koen. Indian J Pharm Sci 75 (1): 83-88.

Vijayalakshmi MA, Sarada NC. 2008. Screening of Costus speciosus extracts for antioxidant activity. Fitoterapia 79 (3): 197-198.

Wang M, Sun GB, Du YY, Tian Y, Liao P, Liu XS, Ye JX, Sun XB. 2017. Myricitrin protects cardiomyocytes from hypoxia/ reoxygenation injury: involvement of heat shock protein 90. Front Pharmacol 8: 353. DOI: 10.3389/fphar.2017.00353.

Yang G, Fang Z, Liu Y, Zhang H, Shi X, Ji Q, Lin Q, Lin R. 2011. Protective effects of chinese traditional medicine buyang huanwu decoction on myocardial injury. Evid Based Compl Alternat Med 2011: 930324. DOI: 10.1093/ecam/nep013. 